\title{
Fate of the Original Retrieval Cues Following the Transfer of Memory in Rats
}

\author{
J.F. Briggs* and D.C. Riccio \\ Department of Psychology, Kent State University, Kent OH, USA
}

\begin{abstract}
Exposure to contextual cues immediately after conditioning enables the new stimuli to retrieve the target memory. But what is the fate of the original cues after this type of transfer of properties? Have they been supplanted by the new cues, or are both sets of stimuli now effective? To address this issue, an experiment was conducted investigating the effectiveness of the original training cues following the transfer of retrieval cues to a new context. Rats were exposed to contextual cues different from training immediately after learning a punishment task. Subjects tested in the new context treated the context as if it were the original, i.e., retrieval cues were transferred to the shifted context. In addition, this transfer had no effect on the original memory as rats that were tested in the original context behaved similarly to those tested in the shifted context. It appears that this transfer of retrieval cues is not a case of erase-and-update, but rather the cues remain for the original context and also become associated with the new context where exposure took place.
\end{abstract}

Key Words: Retrieval cues, context, transfer of memory, passive avoidance, rat.

\section{INTRODUCTION}

The malleability of a memory by post event information has been of interest to researchers for many years [1] and numerous studies have shown that a variety of manipulations can distort memory for a target episode [2]. In contrast, very little research has been directed toward examining changes in the role of retrieval cues. For example, could the cues of a particular learning event gain retrieval control in a different situation? Among the few studies in this area are several from the Rovee-Collier lab. Using human infants as subjects, Rovee-Collier and colleagues have shown that retrieval cues could be transferred to a new environmental context. Taking advantage of the observation that impaired performance is commonly found when subjects are tested in a context different from that used at training (i.e., the context shift effect; see [3-6]), Boller and Rovee-Collier [7] trained 6 month old infants to kick a leg to activate a mobile. Immediately following training, the infants were exposed to a novel context (crib liner). At testing, the infants responded in the new context as if it had been the training context. Thus, the memory for the original training was apparently encoded into the new memory.

Rovee-Collier and colleagues later demonstrated a similar transfer of cues effect using a retroactive interference paradigm [8], and found also that two cues that were not presented together, but were simultaneously activated in memory could become associated [9, see also 10].

To determine whether a memory representation in nonhuman animals could also become encoded or transferred to a new context, Briggs, Fitz, and Riccio [11] recently investi-

*Address correspondence to this author at the Navy Experimental Diving Unit, 321 Bullfinch Road, Panama City, FL 32407, USA; Tel: (850) 2303102; Fax: (850) 230-3197; E-mail: jbriggs @ kent.edu gated whether neutral contextual stimuli could gain retrieval control if presented shortly after learning. Rats received punishment training in a particular context. Immediately following training, some subjects were exposed to a context that differed from that of training. When later tested back in the shifted context these rats showed less impairment of performance (i.e., less of a context shift effect) than nonexposed controls or those that received the exposure after a long delay interval. Thus, the authors concluded that by exposing the animals to the shifted context while the original training memory was still in an active state the retrieval cues associated with training became encoded with the new environmental contextual retrieval cues. A second experiment demonstrated that the transfer of cues was specific to those cues present at the time of exposure, as an exposure to an irrelevant context following training had no beneficial effect on performance. That is, the exposure to irrelevant stimuli failed to enhance retrieval in the test context. These findings showing transfer of retrieval cues to a neutral context were later extended to include an old memory that had been reactivated by cueing [12].

An intriguing aspect of the Boller and Rovee-Collier [7] finding is that the original training context lost its effectiveness as a retrieval cue. The authors suggested that the outcome was due to recoding of an active memory in terms of the contemporary context rather than the displacement of the original context. Interestingly, a similar interpretation has been proposed for a very different phenomenon, experimentally induced retrograde amnesia. ${ }^{1}$ Hinderliter, Webster, and Riccio [13] suggested that the processing of a learning episode becomes encoded in the immediate internal context

${ }^{1}$ We thank Dr. Charles Hinderliter for calling this relationship to our attention. 
produced by the amnestic agent, and the absence of that context at testing leads to a retrieval failure, i.e., retrograde memory impairment. That retrieval based view has since been elaborated and also has been extended to include old reactivated memory, as well as new learning [14-17; see also $18]$.

The issue of recoding (or encoding) of information with new retrieval cues is an important one, but information on its generality is needed. In the Briggs et al. study [11], the new context acquired the ability to retrieve the target memory. However, that experiment did not determine whether the transfer of retrieval to a second context impaired the retrieval capability of the original context, as in the Boller and RoveeCollier [7] study. Accordingly, in the following experiment we asked about the "fate" of the original retrieval cues, using procedures similar to those described for the Briggs et al. [11] research. In brief, this experiment explored the effectiveness of the original memory by testing the subjects back in the original context following the transfer of cues. To insure that the transfer effect on the original memory was successful we evaluated the tendency for performance to be improved when the training and test contexts differed, i.e., alleviation of the context shift effect described above. If retrieval cues become associated with the new context, the context shift effect should be reduced. Moreover, if the original retrieval cues are still intact there should be little disruptive effect caused by testing a separate group in the original context.

\section{METHOD}

\section{Subjects}

The subjects were 32 Long-Evans rats, approximately 85 days of age. The animals were individually housed and were maintained on a 15/9 hr light/dark cycle. Food and water was available ad libitum throughout the course of the experiment. All procedures were conducted in a facility accredited by the Association for Assessment and Accreditation of Laboratory Animal Care in accordance with a protocol approved by Kent State University's Institutional Animal Care and Use Committee.

\section{Apparatus and Contexts}

Training and testing were conducted in two identical 43 X 18 X $18 \mathrm{~cm}$ black-white shuttle boxes with grid floors. Each shuttle box was divided into two compartments of equal size by a guillotine door. The exposure chamber was a 22 X 22 X $24 \mathrm{~cm}$ box made of clear Plexiglas walls and lid. The chamber was placed near the training/testing shuttle box in each context during exposure.

The two shuttle boxes were located in separate rooms that served as contexts. Context A was a $1.62 \times 2.33 \mathrm{~m}$ room with white walls and scented with Airwick Wizard® air freshener with Country Berries ${ }^{\circledR}$ scented oil. White noise $(76 \mathrm{~dB})$ was presented at all times in this context. The room was illuminated by a $25-\mathrm{W}$ red light bulb above the shuttle box. Context B was a brightly lit 1.83 X 2.74 m room with white walls. Posters were placed on each wall to provide visual cues. This room was illuminated by fluorescent houselights. The context was not artificially scented, and no white noise was present.

\section{Procedure}

Prior to the beginning of the experiment, all subjects were handled for two minutes on three consecutive days. Eight rats, randomly assigned to one of 4 conditions, received a single fear conditioning trial in either Context A or Context B. Assignment to the contexts was counterbalanced such that within each group 4 rats were trained in Context A and 4 in Context B. For simplicity of exposition, we refer to context shifts (A to B) generically, regardless of the actual context used.

At training, the rat was brought into the context on the experimenter's arm and remained there for $15 \mathrm{sec}$ to provide brief exposure to the context. The rat was then placed in the white compartment of the shuttle box facing away from the closed guillotine door. After $15 \mathrm{sec}$, the guillotine door was raised and the latency to cross into the black compartment (all four paws) was recorded. The door was then lowered and two inescapable footshocks ( $1 \mathrm{sec}, 0.5 \mathrm{~mA}$ ) were delivered 5 sec and $10 \mathrm{sec}$ after the door was lowered. Five sec after the last footshock, the animal was removed.

Immediately following training, two experimental groups (Transfer and Original) received exposure to the context that differed from training. Exposure consisted of bringing the rat into the shifted context and immediately placing the animal in the white compartment of the shuttle box in an attempt to maintain the activity of the newly acquired information. After $15 \mathrm{sec}$ of exposure to the white side, the rat was removed and placed in the exposure chamber for $4 \mathrm{~min} 45 \mathrm{sec}$. Following the $5 \mathrm{~min}$ exposure treatment, the rat was returned to its home cage. Two additional groups (Same and Shift) did not receive the exposure treatment in the shifted context.

Twenty-four hours after training, all groups were tested. Groups Same and Original were tested in the training context, while groups Shift and Transfer received their test in the shifted context. Testing consisted of one $10 \mathrm{~min}$ passiveavoidance session identical to the training trial, except that no shocks were delivered and the guillotine door remained open. During the testing the rat was placed on the white side and allowed to choose between the white and black compartments. The cross-through latency and total time spent on the safe (white) side (TTS) were recorded as the dependent measures. As the patterns of results were generally similar on both measures, only TTS scores are reported.

\section{RESULTS}

\section{Training}

Rats in all four groups exhibited short cross latencies at training with group means ranging from $12.6 \mathrm{sec}$ to $18.6 \mathrm{sec}$. An analysis of variance (ANOVA) performed on training cross latencies revealed no differences between the four groups $(F(3,28)=.75, p>.50)$.

\section{Counterbalancing}

There were no differences between training cross latencies, cross latencies at test, and TTS in either context. Accordingly, the contexts were collapsed within each group for all further analyses. 


\section{Testing}

Fig. (1) shows the mean TTS scores for all four groups at test. An ANOVA revealed the groups differed significantly $(F(3,28)=7.6, p<.01)$. Tukey's honestly significantly difference (HSD) post-hoc tests were conducted to compare group differences.

As can be seen, a context shift was obtained with the Same group showing significantly more avoidance (long TTS) than the Shift group $(p<.01)$. Thus, being trained and tested in a context different from training impairs spatial avoidance performance. The shift effect was alleviated by the group (Transfer) that was tested in the shifted context where exposure took place, as this group showed as much avoidance as the Same group $(p>.10)$ and more spatial avoidance than the Shift group $(p<.05)$. These results strongly suggest that contextual retrieval cues can be transferred to a new context. Similarly, the group that was tested back in the original training context (Original) also showed as much spatial avoidance as both the Same group and the Transfer group $(p s>.50)$ and significantly more avoidance than the Shift group $(p<.01)$.

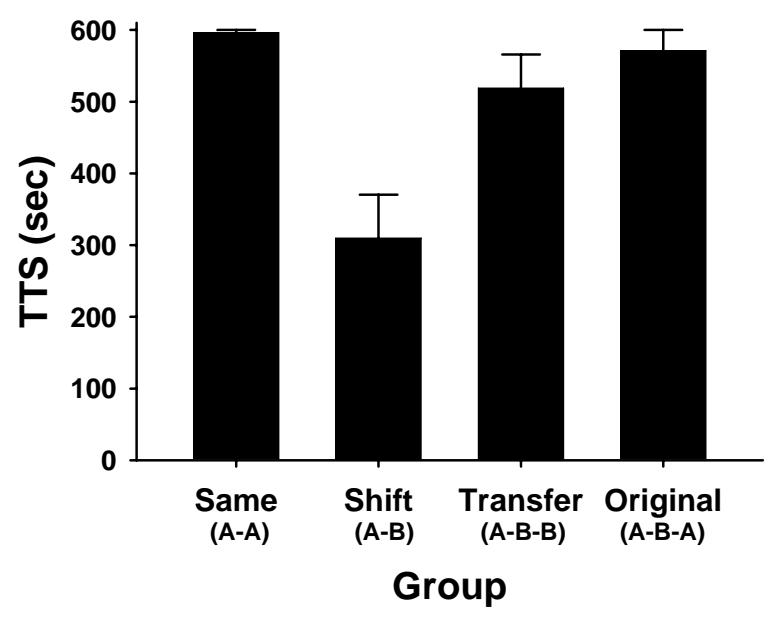

Fig. (1). Mean total time spent on the safe (white) side in seconds for all groups. Error bars represent standard errors of the means. Same and Shift groups represent a context shift effect. The Transfer group shows being exposed to the shifted context immediately following training alleviated the context shift effect. Group Original demonstrates exposure to the shifted context had no effect on the original contextual retrieval cues.

\section{DISCUSSION}

Consistent with other findings [7, 8, 11, see also 12] this experiment demonstrated that retrieval cues could become associated with a new environment by mere exposure to different contextual cues immediately after training. Moreover, the transfer of retrieval cues had no effect on retrieval of memory in the original training context. The same exposure that improved performance in the context shift condition had no effect on the avoidance for the group tested in the original training context. Thus, it appears that this transfer of retrieval cues is not a case of erase-and-update, but rather the cues associated with the original context also become associated with the shifted context where exposure took place.
That the memory representation of the original training cues was not impaired following exposure in the present experiment is inconsistent with other reports. Boller and Rovee-Collier [7] described a loss of effectiveness of the original retrieval cues following a brief exposure to a shifted context. An explanation provided by the authors is that the original memory representation was replaced by the exposure treatment. As stated earlier, Hinderliter and colleagues [13] provide a similar retrieval-based explanation for experimentally induced retrograde amnesia. In their view, the amnestic agent administered during memory encoding produces a specific internal context in which the retrieval cues are represented. When tested later the absence of that unusual internal context leads to a retrieval failure produced by the mismatch of internal cues. Both explanations describe a recoding of the contextual retrieval cues that represents the original learning event.

The fact that in the present experiment the original memory representation remained intact leads to an intriguing question - why in some situations but not in others does exposure to new contextual cues immediately following learning affect the original retrieval cues? One explanation could be the salience of the target event. In addition to the obvious species difference, a major difference between the current investigation and the Boller and Rovee-Collier [7] study is that we used an aversive event in conditioning. This fearbased memory encoded in the rats may be more salient and more significant for survival. Thus, the fear-motivated task could have allowed the original retrieval cues to be protected from modification, allowing the original retrieval cues to be reactivated when tested back in the original context following exposure in the current study. The transfer of retrieval cues and the effectiveness of the original retrieval cues following transfer could be examined with rats using an appetitive task.

A variation on the salience of the training event influencing the effectiveness of the original contextual cues is the delay between training/exposure and testing. The 24-hour retention interval may not have been sufficient to produce a disruption of the original retrieval cues using an aversive event. Testing the effectiveness of the original cues using a longer delay following exposure would demonstrate whether a temporal factor has an effect on the results. However, it seems improbable that the representations of the two contexts would be forgotten at different rates given that the cues of the original context appear to become associated with those of the context where exposure took place.

The salience of contexts could explain the discrepancy between forgetting caused by altering the original memory representation through inducing retrograde amnesia and the lack of modification following the transfer of retrieval cues. Amnestic treatments typically used to induce retrograde amnesia (i.e., electroconvulsive shock, hypothermia, protein synthesis inhibitors) are significant events, which produce salient internal contexts. When testing for effectiveness of original retrieval cues, the mismatch in internal contexts is different enough to cause the forgetting. However, an interesting finding is that retrograde amnesia can be alleviated (i.e., the "forgotten" information can be reactivated) by reexposure to the amnestic agent [13, 18-21]. The notion is that the re-exposure to the internal contextual cues reinstates 
the memory representation caused by the mismatch of internal contexts. Thus, although the exposure treatment used in the current study increased the range of available retrieval cues, it did not do so at the expense of the training cues. Reexposure to the original context still permitted those retrieval cues to reactivate the memory.

Although the present study and previous findings suggest that retrieval cues can be transferred to new situations, there is little information on the nature of the processes involved. The results presented here suggesting that, in this situation, the original representation was not altered emphasize the importance of determining the mechanisms governing the transfer of retrieval cues.

\section{ACKNOWLEDGEMENTS}

Funding of this research was provided by NIMH Grant No. 37535 to David C. Riccio.

\section{REFERENCES}

[1] Roediger HL, McDermott KB. Distortions of memory. In: Tulving E, Craik FIM, Eds. Oxford Handbook of Memory. Oxford: Oxford University Press 2000; pp. 149-62.

[2] Spear NE, Riccio DC. Memory: Phenomena and principles. Boston: Allyn and Bacon 1994.

[3] Godden DR, Baddeley AD. Context-dependent memory in two natural environments: On land and underwater. Brit J Psychol 1975; 66: 325-31

[4] Gordon WC, McCracken KM, Dess-Beech N, Mowrer RR. Mechanisms for the cueing phenomenon: The addition of the cueing context to the training memory. Learn Motiv 1981; 12: 196-211.

[5] Smith SM. Remembering in and out of context. J Exp Psychol Hum Learn Mem 1979; 5: 460-71.

[6] Zhou Y, Riccio DC. Manipulation of components of context: The context shift effect and forgetting of stimulus attributes. Learn Motiv 1996; 27: 400-07.
[7] Boller K, Rovee-Collier C. Contextual coding and recoding of infants' memories. J Exp Child Psychol 1992; 53: 1-23.

[8] Rossi-George A, Rovee-Collier C. Retroactive interference in 3month-old infants. Dev Psychobiol 1999; 35: 167-77.

[9] Cuevas K, Rovee-Collier C, Learmonth AE. Infants form associations between memory representations of stimuli that are absent. Psychol Sci 2006; 17: 543-49.

[10] Dwyer DM, Mackintosh NJ, Boakes RA. Simultaneous activation of the representations of absent cues results in the formation of an excitatory association between them. J Exp Psychol Anim B 1998; 24: 163-71.

[11] Briggs JF, Fitz KI, Riccio DC. Transfer of memory retrieval cues in rats. Psychon B Rev 2007; 14: 495-99.

[12] Briggs JF, Riccio DC. Transfer of old 'reactivated' memory retrieval cues in rats. Learn Motiv 2008; 39: 13-23.

[13] Hinderliter CF, Webster T, Riccio DC. Amnesia induced by hypothermia as a function of treatment-test interval and recooling in rats. Anim Lear Behav 1975; 3: 257-63.

[14] Millin PM, Moody EW, Riccio DC. Interpretations of retrograde amnesia: Old problems redux. Nature Rev Neurosci 2001; 2: 68-70.

[15] Riccio DC, Millin PM, Bogart AR. Reconsolidation: A brief history, a retrieval view, and some recent issues. Learn Memory 2006; 13: $536-44$

[16] Riccio DC, Millin PM, Gisquet-Verrier P. Retrograde amnesia: Forgetting back. Curr Dir Psychol Sci 2003; 12: 41-44.

[17] Riccio DC, Moody EW, Millin PM. Reconsolidation reconsidered. Integr Phys Beh Sci 2002; 37: 245-53.

[18] Briggs JF, Riccio DC. Retrograde amnesia for extinction: Similarities with amnesia for original acquisition memories. Learn Behav 2007; 35: 131-40.

[19] Bradley PM, Galal KM. State-dependent recall can be induced by protein synthesis inhibition: Behavioral and morphological observations. Dev Brain Res 1988; 40: 243-51.

[20] Mactutus CF, Riccio DC. Hypothermia-induced retrograde amnesia: Role of body temperature in memory retrieval. Physiol Psychol 1978; 6: 18-22.

[21] Thompson CI, Neely JE. Dissociated learning in rats produced by electroconvulsive shock. Physiol Behav 1970; 5: 783-86. 